\title{
MINIMIZERS THAT ARE NOT ALSO RELAXED MINIMIZERS*
}

\author{
M. PALLADINO ${ }^{\dagger}$ AND R. B. VINTER $^{\dagger}$ \\ In Memory of Jack Warga
}

\begin{abstract}
Relaxation is a widely used regularization procedure in optimal control, involving the replacement of velocity sets by their convex hulls, to ensure the existence of a minimizer. It can be an important step in the construction of suboptimal controls for the original, unrelaxed, optimal control problem (which may not have a minimizer), based on obtaining a minimizer for the relaxed problem and approximating it. In some cases the infimum cost of the unrelaxed problem is strictly greater than the infimum cost over relaxed state trajectories; we need to identify such situations because then the above procedure fails. The noncoincidence of these two infima leads also to a breakdown of the dynamic programming method because, typically, solving the Hamilton-Jacobi equation yields the minimum cost of the relaxed, not the original, optimal control problem. Following on from earlier work by Warga, we explore the relation between, on the one hand, noncoincidence of the minimum cost of the optimal control and its relaxation and, on the other, abnormality of necessary conditions (in the sense that they take a degenerate form in which the cost multiplier is set to zero). Two kinds of theorems are proved, depending on whether we focus attention on minimizers of the unrelaxed or the relaxed formulation of the optimal control problem. One kind asserts that a local minimizer which is not also a relaxed local minimizer satisfies an abnormal form of the Hamiltonian inclusion. The other asserts that a relaxed local minimizer that is not also a local minimizer also satisfies an abnormal form of Hamiltonian inclusion.
\end{abstract}

Key words. necessary conditions, optimal control, differential inclusions, state constraints

AMS subject classifications. $49 \mathrm{~N}, 49 \mathrm{~K}$

DOI. $10.1137 / 130909627$

1. Introduction. Consider the optimal control problem

$$
\left\{\begin{array}{l}
\text { minimize } g(x(0), x(1)) \\
\text { over absolutely continuous functions } x(.):[0,1] \rightarrow \mathbb{R}^{n} \text { s.t. } \\
\dot{x}(t) \in F(t, x(t)) \text { a.e., } \\
(x(0), x(1)) \in C
\end{array}\right.
$$

the data for which comprise $g: \mathbb{R}^{n} \times \mathbb{R}^{n} \rightarrow \mathbb{R}$, a closed set $C \subset \mathbb{R}^{n} \times \mathbb{R}^{n}$, and a multifunction $F(.,):.[0,1] \times \mathbb{R}^{n} \leadsto \mathbb{R}^{n}$.

A state trajectory $x($.$) is an absolutely continuous function that satisfies \dot{x}(t) \in$ $F(t, x(t))$ a.e. The state trajectory $x($.$) is said to be feasible if (x(0), x(1)) \in C$.

Let $\left(X,\|.\|_{X}\right)$ be a normed space of absolutely continuous functions $x():.[0,1] \rightarrow$ $\mathbb{R}^{n}$. We say that the state trajectory $\bar{x}($.$) is a minimizer if it achieves the minimum of$ $g(x(0), x(1))$ over all feasible state trajectories $x($.$) . It is called an X$-local minimizer if, for some $\epsilon>0$,

$$
g(x(0), x(1)) \geq g(\bar{x}(0), \bar{x}(1))
$$

*Received by the editors February 14, 2013; accepted for publication (in revised form) April 28, 2014; published electronically July 10, 2014. This work was cofunded by the European Union under the 7th Framework Programme FP7-PEOPLE-2010-ITN, grant agreement 264735-SADCO and by EPSRC, grant EP/G066477/1.

http://www.siam.org/journals/sicon/52-4/90962.html

$\dagger$ Department of Electrical and Electronic Engineering, Imperial College London, Exhibition Road, London, SW7 2BT, UK (m.palladino@imperial.ac.uk, r.vinter@imperial.ac.uk). 
for all feasible state trajectories $x($.$) such that$

$$
\|x(.)-\bar{x}(.)\|_{X} \leq \epsilon .
$$

The norm $\|.\|_{X}$ of principal interest in this paper is the $L^{\infty}$ norm. Consistent with standard terminology, an $L^{\infty}$ local minimizer is also called a strong local minimizer. But the $W^{1,1}$ norm will also be considered, where

$$
\|x(.)\|_{W^{1,1}}=|x(0)|+\int_{0}^{1}|\dot{x}(s)| d s .
$$

Now consider the reachable set $\mathcal{R}$ which, here, we take to be the set of all possible endpoint values of state trajectories:

$$
\mathcal{R}:=\{(x(0), x(1)) \mid x(.) \text { is a state trajectory }\} .
$$

The problem $(P)$ can be reformulated in terms of $\mathcal{R}$ :

$$
\text { minimize } g(x(0), x(1)) \text { over }(x(0), x(1)) \in \mathcal{R} \cap C .
$$

A standard framework for studying optimal control problems, in which the existence of minimizing feasible trajectories is guaranteed, is to impose a hypothesis regarding convexity of the velocity sets:

$$
(\mathrm{C}): F(t, x) \text { is convex for all }(t, x) \in[0,1] \times \mathbb{R}^{n} \text {. }
$$

For then we can show (under additional, unrestrictive, hypotheses), by means of a weak sequential compactness analysis, that the set $\mathcal{R}$ is closed. It follows that if, also, the set $\mathcal{R} \cap C$ is bounded and nonempty, and $g$ is continuous, then $(P)$ automatically has a minimizing feasible $F$-trajectory, as the minimizer of a continuous function over a nonempty compact set.

Suppose, on the other hand, that the convexity hypothesis is violated. A standard regularization procedure, aimed at enlarging the domain of $(P)$, in order to guarantee the existence of minimizers, is to consider the relaxed problem

$$
\left\{\begin{array}{l}
\text { minimize } g(x(0), x(1)) \\
\text { over absolutely continuous functions } x(.):[0,1] \rightarrow \mathbb{R}^{n} \text { such that } \\
\dot{x}(t) \in \operatorname{co} F(t, x(t)) \text { a.e., } \\
(x(0), x(1)) \in C .
\end{array}\right.
$$

State trajectories and strong local minimizers for $(R)$ are referred to as "relaxed state trajectories" and "relaxed strong local minimizers," respectively. Since the velocity sets $F(t, x)$ have been replaced by their convex hulls, the relaxed problem automatically has a minimizer (under additional, mild hypotheses).

The relaxation theorem (see, e.g., [9, Thm. 2.7.1]) asserts (under appropriate, unrestrictive, hypotheses) that a relaxed state trajectory $x($.$) can be approximated$ arbitrarily closely w.r.t. the $L^{\infty}$ norm by a state trajectory. It follows that, if we define $\mathcal{R}_{\text {relaxed }}$ to be the reachable set for state trajectories associated with $(R)$, then

$$
\mathcal{R}_{\text {relaxed }}=\overline{\mathcal{R}},
$$

where $\overline{\mathcal{R}}$ is the closure of $\mathcal{R}$. Often

$$
\begin{aligned}
\inf \{g(x(0), x(1)) \mid & (x(0), x(1)) \in \mathcal{R} \cap C\} \\
& =\inf \{g(x(0), x(1)) \mid(x(0), x(1)) \in \overline{\mathcal{R}} \cap C\} .
\end{aligned}
$$

Copyright $@$ ( ) by SIAM. Unauthorized reproduction of this article is prohibited. 


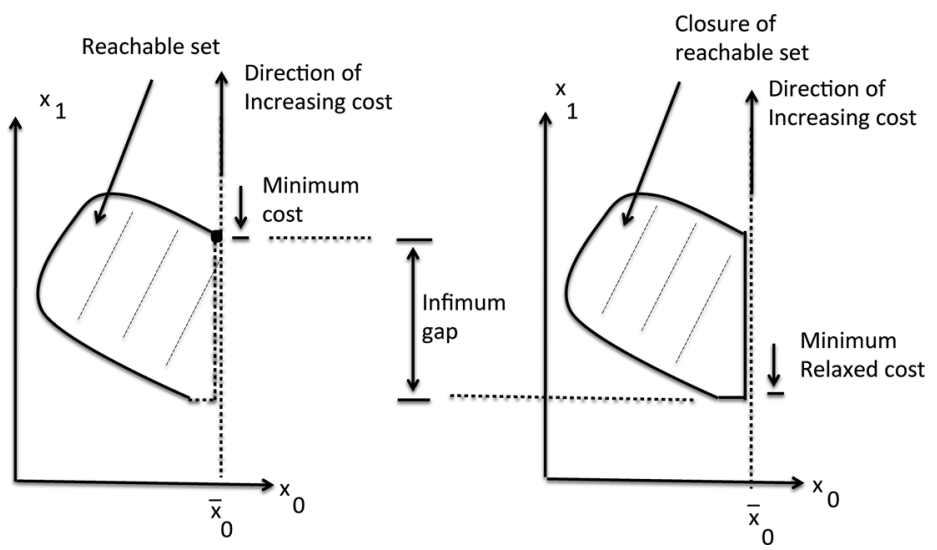

FIG. 1. Noncoinciding infima.

It may arise however that

$$
\begin{aligned}
\inf \{g(x(0), x(1)) \mid & (x(0), x(1)) \in \mathcal{R} \cap C\} \\
& >\inf \{g(x(0), x(1)) \mid(x(0), x(1)) \in \overline{\mathcal{R}} \cap C\} .
\end{aligned}
$$

This can be equivalently written

$$
\inf (R)<\inf (P),
$$

where $\inf (R)$ and $\inf (P)$ denote the infimum costs of $(R)$ and $(P)$, respectively.

The phenomenon is illustrated by Figure 1, in which the state dimension $n=1$, $C=\left\{\bar{x}_{0}\right\} \times \mathbb{R}$, and $g\left(x_{0}, x_{1}\right)=x_{1}$. The figure shows a reachable set and its closure, obtained by adding a vertical line segment. The infimum cost for $(P)$ (respectively, $(R))$ is the lowest intersection of the vertical line through $\bar{x}_{0}$ with the reachable set (respectively, the closure of the reachable set). These minimum costs differ because there exist lower points in the intersection with the closure of the reachable set than with the reachable set itself.

It is important to identify the occurrence of an "infimum gap" (1.1) (between the problem $(P)$ and its relaxation $(R)$ ) for several reasons. First we note that the Hamilton-Jacobi equation is the same for $(P)$ and $(R)$, but in circumstances when there is a unique generalized solution to the Hamilton-Jacobi equation, this solution coincides with the value function of the relaxed problem; the dynamic programming approach fails to provide the value function for $(P)$ in this case; see [9, Thm. 12.3.7]. Second, the presence of an infimum gap indicates instability of the minimum cost and of the set of optimal processes under perturbations to the endpoint constraint, which add to the difficulty of numerical solution. Indeed, normality (which, as we shall see, is a sufficient condition for absence of an infimum gap) is cited as a hypothesis, in the analysis for numerical solution techniques with guaranteed convergence [1].

This paper is concerned with the link between the existence of an infimum gap, at least in a local sense, and the degeneracy of the necessary conditions of optimality, expressed in terms of Clarke's Hamiltonian inclusion. This necessary condition of optimality will be discussed shortly. Two consequences of an infimum gap are explored, which differ according to whether we focus attention on a strong local minimizer which cannot be interpreted as a relaxed strong local minimizer, or on a relaxed min- 
imizer, whose cost is strictly less than the infimum cost over admissible (nonrelaxed) processes.

Type A. A strong local minimizer satisfies necessary conditions of optimality in abnormal form (i.e., with cost multiplier zero) if it is not also a relaxed strong local minimizer.

Type B. A relaxed strong local minimizer satisfies necessary conditions of optimality (for the relaxed problem) in abnormal form if its cost is strictly less than the infimum cost over all feasible processes, whose state trajectories are close (in the $L^{\infty}$ sense) to that of the relaxed strong local minimizer.

Warga pioneered investigations into the link between the existence of an infimum gap and validity of first order necessary conditions in abnormal form. The link has also been studied by Ioffe [5].

In his monograph [11] Warga proved a Type B relation for optimal control problems with state constraints, in which the dynamic constraint is expressed as a controlled differential equation and the set of necessary conditions considered is the Pontryagin maximum principle. (The expository paper [10] stresses the contrapositive interpretation "if there are no relaxed state trajectories satisfying a relaxed version of the Pontryagin Maximum Priniciple with cost multiplier $\lambda=0$, then there cannot exist minimizers that are not also relaxed minimizers"). In a subsequent paper [12], Warga generalized his earlier Type B results to allow for nonsmooth data, making use of the local approximations based on "derivative containers," developed in [13].

Type A relations have received less attention, surprisingly so since they come closer to addressing the key underlying question, when is a minimizer not a relaxed minimizer? We comment below on a Type A relationship asserted by Ioffe [5].

In this paper we prove, for the first time, a Type B relation for optimal control problems in which the dynamic constraint is formulated as a differential inclusion. We also give the first proof of a Type A relation for such problems, when "relaxed local minimizer" is interpreted in the standard, $L^{\infty}$, sense, and observe that earlier claims of such a relation in which "local" is interpreted w.r.t. a different topology are not, in general, correct. The relations are proved for problems with (pathwise) state constraints. But they are initially stated for state constraint-free problems, out of concern that the simple relations between the existence of "infimum gap" and abnormality of multipliers not be obscured, initially, by the extra technical machinery required to take account of state constraints.

The following notation will be used throughout the paper. For vectors $x \in \mathbb{R}^{n}$, $|x|$ denotes the Euclidean length. $B$ denotes the closed unit ball in $\mathbb{R}^{n}$. Given a multifunction $\Gamma():. \mathbb{R}^{n} \leadsto \mathbb{R}^{k}$, the graph of $\Gamma($.$) , written \operatorname{Gr}\{\Gamma()$.$\} , is the set$ $\left\{(x, v) \in \mathbb{R}^{n} \times \mathbb{R}^{k} \mid v \in \Gamma(x)\right\}$. Given a set $A \subset \mathbb{R}^{n}$ and a point $x \in \mathbb{R}^{n}$, we denote by $d_{A}(x)$ the Euclidean distance of a point $x \in \mathbb{R}^{n}$ from $A$ :

$$
d_{A}(x):=\inf \{|x-y| \mid y \in A\} .
$$

$W^{1,1}\left([0,1] ; \mathbb{R}^{n}\right)$ is the space of absolutely continuous $\mathbb{R}^{n}$-valued functions on $[0,1]$. We write $W^{1,1}$ in place of $W^{1,1}\left([0,1] ; \mathbb{R}^{n}\right)$, etc. when the meaning is clear. We denote by $N B V^{+}[0,1]$ the space of increasing, real-valued functions $\mu($.$) on [0,1]$ of bounded variation, vanishing at the point 0 and right continuous on $(0,1)$. The total variation of a function $\mu(.) \in N B V^{+}[0,1]$ is written $\|\mu\|_{\mathrm{TV}}$. As is well known, each point $\mu(.) \in N B V^{+}[0,1]$ defines a Borel measure on $[0,1]$. This associated measure is also denoted $\mu$. 
We shall use several constructs of nonsmooth analysis. Given a closed set $D \subset \mathbb{R}^{k}$ and a point $\bar{x} \in D$, the limiting normal cone $N_{D}(\bar{x})$ of $D$ at $\bar{x}$ is defined to be

$N_{D}(\bar{x}):=\left\{p \mid \exists x_{i} \stackrel{D}{\longrightarrow} \bar{x}, p_{i} \longrightarrow p\right.$ s.t. $\quad \limsup _{x \stackrel{D}{\rightarrow} x_{i}} \frac{p_{i} \cdot\left(x-x_{i}\right)}{\left|x-x_{i}\right|} \leq 0 \quad$ for all $\left.i \in \mathbb{N}\right\}$.

Here, the notation $y^{\prime} \stackrel{D}{\rightarrow} y$ is employed to indicate that all points in the convergent sequence $\left\{y^{\prime}\right\}$ lie in $D$.

Take a lower semicontinuous function $f: \mathbb{R}^{k} \rightarrow \mathbb{R} \cup\{+\infty\}$ and a point $\bar{x} \in$ $\operatorname{dom} f:=\left\{x \in \mathbb{R}^{k} \mid f(x)<+\infty\right\}$. The limiting subdifferential of $f$ at $\bar{x}$ (termed the subdifferential in [7], [9]) is denoted $\partial f(\bar{x})$ :

$$
\begin{aligned}
\partial f(\bar{x}):= & \left\{\xi \mid \exists \xi_{i} \rightarrow \xi \text { and } x_{i} \stackrel{\operatorname{dom} f}{\longrightarrow} \bar{x}\right. \text { such that } \\
& \left.\limsup _{x \rightarrow x_{i}} \frac{\xi_{i} \cdot\left(x-x_{i}\right)-\varphi(x)+\varphi\left(x_{i}\right)}{\left|x-x_{i}\right|} \leq 0 \text { for all } i \in \mathbb{N}\right\} .
\end{aligned}
$$

For further information regarding these objects, we refer to [4], [7], or [9].

2. Conditions for noncoincidence of infima. In this section we state two theorems relating the existence of a gap (in some local sense) between the infimum costs for the optimal control problem $(P)$ and its relaxed counterpart $(R)$, and the validity of the Hamiltonian inclusion in abnormal form. The following hypotheses, in which $\bar{x}($.$) is a given absolutely continuous function, will be invoked.$

(H1). $F(., x)$ is an $\mathcal{L}$-measurable multifunction for each $x$ and $F(.,$.$) takes values$ of closed sets.

(H2). There exist $k(),. c(.) \in L^{1}$ and $\bar{\epsilon}>0$ such that

$$
\begin{aligned}
F(t, x) & \subset F\left(t, x^{\prime}\right)+k(t)\left(\left|x-x^{\prime}\right|\right) B \text { and } \\
F(t, x) & \in c(t) B \\
\text { for all } x, x^{\prime} & \in \bar{x}(t)+\bar{\epsilon} B \text { a.e. } t \in[0,1] .
\end{aligned}
$$

Define the Hamiltonian function $H(., .,):.[0,1] \times \mathbb{R}^{n} \times \mathbb{R}^{n} \rightarrow \mathbb{R}$

$$
H(t, x, p):=\max _{e \in F(t, x)} p \cdot e .
$$

The first theorem is a Type A relation.

TheOREM 2.1. Let $\bar{x}($.$) be a strong local minimizer for problem (P)$. Assume that hypotheses (H1) and (H2) are satisfied and $g(.,$.$) is Lipschitz continuous on a$ neighborhood of $(\bar{x}(0), \bar{x}(1))$.

(a) Then there exist $p(.) \in W^{1,1}\left([0,1] ; \mathbb{R}^{n}\right)$ and $\lambda \geq 0$ such that

(i) $(p(),. \lambda) \neq(0,0)$,

(ii) $(-\dot{p}(t), \dot{\bar{x}}(t)) \in \operatorname{co}_{x, p} H(t, \bar{x}(t), p(t))$ a.e.,

(iii) $(p(0),-p(1)) \in \lambda \partial g(\bar{x}(0), \bar{x}(1))+N_{C}(\bar{x}(0), \bar{x}(1))$.

(b) Suppose that, for every $\epsilon>0$, there exists a feasible relaxed state trajectory $x($.$) such that$

$$
g(\bar{x}(0), \bar{x}(1))>g(x(0), x(1))
$$

and $\|x(.)-\bar{x}(.)\|_{L^{\infty}} \leq \epsilon$, (i.e., $\bar{x}($.$) is not also a relaxed strong local mini-$ mizer).

Then conditions (i)-(iii) above are satisfied for some choice of multipliers $(p(),. \lambda)$, such that $\lambda=0$.

Theorem 2.1 is proved in section 4 .

Copyright $@$ by SIAM. Unauthorized reproduction of this article is prohibited. 
Comments.

(1) Part (a) is Clarke's well-known Hamiltonian inclusion [2]. Interest focuses on part (b), which is a Type A relation.

(2) The contrapositive statement of part (b) is a sufficient condition for the absence of an infimum gap (in a local sense): if $\bar{x}($.$) is a strong local minimizer$ such that, given any multipliers $(p(),. \lambda)$ satisfying conditions (i)-(iii), we have $\lambda \neq 0$, then $\bar{x}($.$) is also a relaxed strong local minimizer.$

(3) Recall the generalized Euler-Lagrange condition, which asserts a modification of the set of conditions (a) of Theorem 2.1 in which condition (a)(ii) is replaced by the stronger condition.

(ii) $^{\prime}-\dot{p}(t) \in \operatorname{co}\left\{r \mid(r, p(t)) \in N_{\operatorname{Gr}\{F(t, .)\}}(\bar{x}(t), \dot{\bar{x}}(t))\right\}$ a.e.

Ioffe [5, Prop. 9],[6] asserts the following Type A relation for differential inclusions: "take a $W^{1,1}$ local minimizer $\bar{x}($.$) which is not also a relaxed W^{1,1}$ local minimizer. Then $\bar{x}($.$) satisfies the generalized Euler-Lagrange inclusion$ for some set of multipliers $(p(),. \lambda)$ in which $\lambda=0 . "$ This assertion is incorrect in general. Reference [8] provides an example of a $W^{1,1}$ local minimizer for a free right endpoint optimal control problem, which is not also a relaxed $W^{1,1}$ local minimizer. The fact that the right endpoint is free in this example means that all multiplier sets have nonzero cost multiplier; in other words, it serves also as a counterexample to the above assertion.

The second theorem includes a Type B relation.

TheOREM 2.2. Let $\bar{x}($.$) be a relaxed feasible state trajectory related to problem$ $(P)$. Assume that hypotheses $(\mathrm{H} 1)$ and $(\mathrm{H} 2)$ are satisfied.

(a) Suppose that $g(.,$.$) is Lipschitz continuous on a neighborhood of (\bar{x}(0), \bar{x}(1))$ and there exists $\epsilon>0$ such that

$$
\begin{array}{r}
g(\bar{x}(0), \bar{x}(1))=\inf \{g(x(0), x(1)) \mid x(.) \text { is a (nonrelaxed) feasible } \\
\text { state trajectory such that } \left.\|x(.)-\bar{x}(.)\|_{L^{\infty}} \leq \epsilon\right\}
\end{array}
$$

Then there exist $p(.) \in W^{1,1}\left([0,1] ; \mathbb{R}^{n}\right)$ and $\lambda \geq 0$ such that

(i) $(p(),. \lambda) \neq(0,0)$,

(ii) $(-\dot{p}(t), \dot{\bar{x}}(t)) \in \operatorname{co}_{x, p} H(t, \bar{x}(t), p(t))$ a.e.,

(iii) $(p(0),-p(1)) \in \lambda \partial g(\bar{x}(0), \bar{x}(1))+N_{C}(\bar{x}(0), \bar{x}(1))$.

(b) Suppose that $g(.,$.$) is continuous on a neighborhood of (\bar{x}(0), \bar{x}(1))$ and, for some $\epsilon>0$,

$$
\begin{array}{r}
g(\bar{x}(0), \bar{x}(1))<\inf \{g(x(0), x(1)) \mid x(.) \text { is a feasible (nonrelaxed) } \\
\text { state trajectory such that } \left.\|x(.)-\bar{x}(.)\|_{L^{\infty}} \leq \epsilon\right\}
\end{array}
$$

Then conditions (i)-(iii) above are satisfied for some choice of multipliers $(p(),. \lambda)$, such that $\lambda=0$.

Comments.

(1) It is well known that if a relaxed feasible state trajectory $\bar{x}($.$) achieves the$ minimum cost over relaxed feasible state trajectories in an $L^{\infty}$ neighborhood about $\bar{x}($.$) , then \bar{x}($.$) satisfies the Hamiltonian inclusion. (This follows from$ applying the Hamiltonian necessary condition to the relaxed problem.) Part (a) of the theorem tells us a little bit more: it says that the Hamiltonian inclusion is satisfied at the relaxed feasible state trajectory $\bar{x}($.$) , if the cost of$ $\bar{x}($.$) coincides with the infimum cost for the problem over the smaller set of$ (nonrelaxed) feasible state trajectories in some $L^{\infty}$ neighborhood about $\bar{x}($.$) ,$

Copyright (c) by SIAM. Unauthorized reproduction of this article is prohibited. 
an apparently new result for optimal control problems formulated in terms of differential inclusions. This remains the case even if the infimum is not attained over (nonrelaxed) feasible state trajectories on some $L^{\infty}$ neighborhood about $\bar{x}($.$) .$

(2) Recall that a Type B relation (involving the Hamiltonian inclusion) asserts that, if a relaxed state trajectory $\bar{x}($.$) which is a strong local minimizer for$ the relaxed problem has cost less than the infimum cost over (nonrelaxed) state trajectories in an $L^{\infty}$ neighborhood about $\bar{x}($.$) , then \bar{x}($.$) satisfies the$ Hamiltonian inclusion in abnormal form. Notice that part (b) of the theorem is a stronger statement because it says the Hamiltonian inclusion is satisfied in abnormal form, even if $\bar{x}($.$) is not a relaxed L^{\infty}$ local minimizer, but merely has cost strictly less than the infimum cost over (nonrelaxed) feasible state trajectories in some $L^{\infty}$ neighborhood about $\bar{x}($.$) . Warga's Type B relations$ for optimal control problems involving a differential equation with control term also include this refinement.

3. An example. In this section we provide an example of an optimal control problem which illustrates the assertions of Theorems 2.1 and 2.2. Earlier examples of optimal control problems, in which the infimum costs over admissible state trajectories and over relaxed feasible state trajectories fail to coincide, are to be found, for example, in [11, p. 246]:

$$
\left\{\begin{array}{l}
\operatorname{minimize}-x_{1}(1) \\
\text { over }\left(x(.)=\left(x_{1}(.), x_{2}(.), x_{3}(.)\right)\right. \text { satisfying } \\
\left(\dot{x}_{1}(t), \dot{x}_{2}(t), \dot{x}_{3}(t)\right) \in\left\{\left(0, x_{1}(t),\left|x_{2}(t)\right|^{2}\right)\right\} \cup\left\{\left(0,-x_{1}(t),\left|x_{2}(t)\right|^{2}\right)\right\} \\
x_{2}(0)=x_{3}(0)=x_{3}(1)=0 .
\end{array}\right.
$$

This is an example of $(P)$, in which $n=3$,

$$
\begin{array}{r}
\left.F\left(t, x_{1}, x_{2}, x_{3}\right)\right)=\left\{\left(0, x_{1}, x_{2}^{2}\right)\right\} \cup\left\{\left(0,-x_{1}, x_{2}^{2}\right)\right\}, \\
\left.g\left(\left(x_{1}^{0}, x_{2}^{0}, x_{3}^{0}\right)\right),\left(x_{1}^{1}, x_{2}^{1}, x_{3}^{1}\right)\right)=-x_{1}^{1}, \text { and } \\
C=(\mathbb{R} \times\{0\} \times\{0\}) \times(\mathbb{R} \times \mathbb{R} \times\{0\}) .
\end{array}
$$

Claim 3.1. $(\bar{x}(.) \equiv(0,0,0))$ is a minimizer for $(E)$.

To validate the claim, suppose there exists a feasible state trajectory $x($.$) with$ cost lower than that of $\bar{x}($.$) . Since \dot{x}_{1}(t)=0$ and the cost is $-x_{1}(1), x_{1} \equiv \alpha$ for some $\alpha>0$. But $\dot{x}_{3}(t)=\left|x_{2}(t)\right|^{2} \geq 0$ and $x_{3}(0)=x_{3}(1)=0$. We deduce from the relation

$$
x_{3}(1)-x_{3}(0)=\int_{0}^{1}\left|x_{2}(t)\right|^{2} d t
$$

that $x_{2}(.) \equiv 0$. It follows that $\dot{x}_{2}(t) \equiv 0$ a.e. However $\dot{x}_{2}(t) \in\{\alpha\} \cup\{-\alpha\}$ a.e. We conclude that $\dot{x}_{2}(t) \neq 0$ a.e.. From this contradiction we deduce that no feasible state trajectory exists with cost less than that of $\bar{x}(.) \equiv(0,0,0)$, as claimed.

Observe that $\bar{x}(.) \equiv(0,0,0)$ is not a relaxed strong local minimizer. This is because, for any $\alpha>0$, the arc

$$
x^{\alpha}(.) \equiv(\alpha, 0,0)
$$

satisfies the convexified dynamic constraint $\dot{x}^{\alpha}(t) \in \operatorname{co} F\left(x^{\alpha}(t)\right)$ a.e., and also the endpoint constraints. It is therefore a feasible relaxed state trajectory. But (by 
adjustment of $\alpha)\left\|x^{\alpha}(.)-\bar{x}(.)\right\|_{L^{\infty}}=\alpha$ can be made arbitrarily small. Yet its cost is $-\alpha$, which is strictly less than that of $\bar{x}($.$) .$

Illustration of Theorem 2.1. We examine the Hamiltonian inclusion condition at $\bar{x}(.) \equiv(0,0,0)$. The Hamiltonian is

$$
H(x, p)=\left|p_{2} x_{1}\right|+p_{3} x_{2}^{2}
$$

and

$$
\partial H(x, p)=\left\{\left(\gamma p_{2}, 2 p_{3} x_{2}, 0,0, \gamma x_{1}, x_{2}^{2}\right) \mid \gamma \in[-1,1]\right\} .
$$

Denote by $(p(),. \lambda)$ the (nontrivial) costate/cost multiplier pair. The Hamiltonian inclusion and transversality conditions take the following form: there exists a measurable function $\gamma():.[0,1] \rightarrow[-1,1]$ such that

$$
\begin{aligned}
-\dot{p}_{1}(t) & =\gamma(t) p_{2}(t),-\dot{p}_{2}(t)=2 p_{3}(t) \bar{x}_{2}(t),-\dot{p}_{3}(t)=0, \\
-\dot{\bar{x}}_{1}(t) & =0,-\dot{\bar{x}}_{2}(t)=\gamma(t) \bar{x}_{1}(t), \dot{\bar{x}}_{3}(t)=\bar{x}_{2}^{2}(t) \\
p_{1}(0) & =0, p_{1}(1)=\lambda, p_{2}(1)=0
\end{aligned}
$$

These conditions (in which $\bar{x}(.) \equiv 0$ ), are satisfied if and only if, for some $k \neq 0$,

$$
p_{1}(.) \equiv 0, p_{2}(.) \equiv 0, p_{3}(.) \equiv k, \text { and } \lambda=0 .
$$

(We can choose $\gamma($.$) to be any measurable function taking values in [-1,+1]$. ) We see that $\bar{x}($.$) satisfies the Hamiltonian condition with cost multiplier \lambda=0$, as predicted by Theorem 2.1. In fact, in this example, all possible choices of multipliers are signed scalings (involving multiplication by the nonzero constant $k$ ) of the multiplier set $(p(.) \equiv(0,0,1), \lambda=0)$.

Illustration of Theorem 2.2. Now fix $\alpha>0$ and consider the feasible relaxed state trajectory $x^{\alpha}(.) \equiv(\alpha, 0,0)$. As we have observed, $x^{\alpha}($.$) has cost strictly lower$ than that of any feasible state trajectory. The Hamiltonian inclusion conditions are again given by (3.1)-(3.3), but now $\bar{x}($.$) is replaced by x^{\alpha}($.$) . The conditions are$ satisfied if and only if $p($.$) and \lambda$ are given by (3.4). (Now, however, we must choose $\gamma(.) \equiv 0$ since $x_{1}^{\alpha}$ is a nonzero value.) We have confirmed that $x^{\alpha}(.) \equiv(\alpha, 0,0)$ satisfies the Hamiltonian inclusion condition with cost multiplier $\lambda=0$, as predicted by Theorem 2.2 .

4. State constraints. Consider now the variant on problem $(P)$,

$$
\left\{\begin{array}{l}
\text { minimize } g(x(0), x(1)) \\
\text { over absolutely continuous functions } x(.):[0,1] \rightarrow \mathbb{R}^{n} \text { s.t. } \\
\dot{x}(t) \in F(t, x(t)) \text { a.e., } \\
h(t, x(t)) \leq 0 \text { for all } t \in[0,1] \\
(x(0), x(1)) \in C
\end{array}\right.
$$

in which an additional, pathwise state constraint

$$
h(t, x(t)) \leq 0 \text { for all } t \in[0,1]
$$

is imposed, expressed in terms of the function $h(.,):.[0,1] \times \mathbb{R}^{n} \rightarrow \mathbb{R}$. Feasible state trajectories, feasible relaxed state trajectories, strong local minimizers, and strong local minimizers for problem $\left(P_{S}\right)$ have similar definitions as those for $(P)$, except the feasible state trajectories in question are now required additionally to satisfy (4.1). 
The related relaxed optimal control problem, which results from replacing $F(t, x)$ by its convex hull, is

$$
\left\{\begin{array}{l}
\text { minimize } g(x(0), x(1)) \\
\text { over absolutely continuous functions } x(.):[0,1] \rightarrow \mathbb{R}^{n} \text { s.t. } \\
\dot{x}(t) \in \operatorname{co} F(t, x(t)) \text { a.e. } \\
h(t, x(t)) \leq 0 \text { for all } t \in[0,1] \\
(x(0), x(1)) \in C .
\end{array}\right.
$$

The following hypothesis, concerning the state constraint functional $h(.,$.$) and the$ $\operatorname{arc} \bar{x}($.$) of interest, will be invoked. Here, \bar{\epsilon}>0$ is as in hypothesis (H2).

(H3). $h(.,$.$) is upper semicontinuous on \left\{(t, x) \in[0,1] \times \mathbb{R}^{n} \mid x \in \bar{x}(t)+\bar{\epsilon} B\right\}$ and there exists a constant $k_{h}$ such that

$$
\left|h(t, x)-h\left(t, x^{\prime}\right)\right| \leq k_{h}\left|x-x^{\prime}\right|
$$

for all $x, x^{\prime} \in \bar{x}(t)+\bar{\epsilon} B$ and $t \in[0,1]$.

A version of the Hamiltonian inclusion conditions is valid at a strong local minimizer $\bar{x}($.$) for \left(P_{S}\right)$. Now, the conditions assert the existence of multipliers $(p(),. \mu(),. \lambda)$ which incorporate not just the costate and cost multiplier $\lambda$, but an extra multiplier $\mu($.$) associated with the state constraint.$

The following two theorems, relating the existence of an infimum gap and validity of the Hamiltonian inclusion conditions with cost multiplier $\lambda=0$, are generalizations to the state constraint setting of Theorems 2.1 and 2.2.

Theorem 4.1. Let $\bar{x}($.$) be a strong local minimizer for \left(P_{S}\right)$. Assume that hypotheses (H1)-(H3) are satisfied and $g(.,$.$) is Lipschitz continuous on a neighborhood$ of $(\bar{x}(0), \bar{x}(1))$.

(a) Then there exist $p(.) \in W^{1,1}\left([0,1] ; \mathbb{R}^{n}\right)$, a function $\mu(.) \in N B V^{+}[0,1]$, a $\mu$-integrable function $m($.$) , and \lambda \geq 0$ such that

(i) $(p(),. \lambda, \mu()) \neq.(0,0,0)$,

(ii) $(-\dot{p}(t), \dot{\bar{x}}(t)) \in \operatorname{co} \partial_{x, p} H\left(t, \bar{x}(t), p(t)+\int_{[0, t]} m(s) \mu(d s)\right)$ a.e.,

(iii) $\left(p(0),-\left(p(1)+\int_{[0,1]} m(s) \mu(d s)\right)\right) \in \lambda \partial g(\bar{x}(0), \bar{x}(1))+N_{C}(\bar{x}(0), \bar{x}(1))$,

(iv) $m(t) \in \partial_{x}^{>} h(t, \bar{x}(t)) \quad \mu$-a.e. $t \in[0,1]$;

(b) Suppose that, for every $\epsilon>0$, there exists a feasible relaxed state trajectory $x($.$) such that$

$$
g(\bar{x}(0), \bar{x}(1))>g(x(0), x(1))
$$

and $\|x(.)-\bar{x}(.)\|_{L^{\infty}} \leq \epsilon$, (i.e., $\bar{x}($.$) is not also a relaxed strong local mini-$ mizer).

Then conditions (i)-(iv) above are satisfied for some choice of multipliers $(p(),. \mu, \lambda)$ such that $\lambda=0$.

Here $\partial_{x}^{>} h(t, x)$ is the set

$$
\begin{aligned}
\partial_{x}^{>} h(t, x):=\left\{\xi \mid \text { there exist } x_{i} \rightarrow x, t_{i} \rightarrow t, \xi_{i} \rightarrow \xi, \text { s.t., for each } i,\right. \\
\\
\left.\quad \nabla_{x} h\left(t_{i}, x_{i}\right) \text { exists, } \xi_{i}=\nabla_{x} h\left(t_{i}, x_{i}\right), \text { and } h\left(t_{i}, x_{i}\right)>0\right\} .
\end{aligned}
$$

The theorem is proved in section 5 .

TheOREm 4.2. Let $\bar{x}($.$) , be a feasible relaxed state trajectory. Assume that hy-$ potheses $(\mathrm{H} 1)-(\mathrm{H} 3)$ are satisfied.

Copyright $@$ by SIAM. Unauthorized reproduction of this article is prohibited. 
(a) Suppose that $g(.,$.$) is Lipschitz continuous on a neighborhood of (\bar{x}(0), \bar{x}(1))$ and there exists $\epsilon>0$ such that

$$
\begin{array}{r}
g(\bar{x}(0), \bar{x}(1))=\inf \{g(x(0), x(1)) \mid x(.) \text { is a feasible (nonrelaxed) } \\
\text { state trajectory such that } \left.\|x(.)-\bar{x}(.)\|_{L^{\infty}} \leq \epsilon\right\} .
\end{array}
$$

Then there exist $p(.) \in W^{1,1}\left([0,1] ; \mathbb{R}^{n}\right), \lambda \geq 0, \mu(.) \in N B V^{+}[0,1]$, and a $\mu$-integrable function $m($.$) such that$

(i) $(p(),. \lambda, \mu()) \neq.(0,0,0)$,

(ii) $(-\dot{p}(t), \dot{\bar{x}}(t)) \in \operatorname{co} \partial_{x, p} H\left(t, \bar{x}(t), p(t)+\int_{[0, t]} m(s) \mu(d s)\right)$ a.e.,

(iii) $\left(p(0),-\left(p(1)+\int_{[0,1]} m(s) \mu(d s)\right)\right) \in \lambda \partial g(\bar{x}(0), \bar{x}(1))+N_{C}(\bar{x}(0), \bar{x}(1))$,

(iv) $m(t) \in \partial_{x}^{>} h(t, \bar{x}(t)) \quad \mu$-a.e. $t \in[0,1]$.

(b) Suppose that $g(.,$.$) is continuous on a neighborhood of (\bar{x}(0), \bar{x}(1))$ and, for some $\epsilon>0$,

$$
\begin{array}{r}
g(\bar{x}(0), \bar{x}(1))<\inf \{g(x(0), x(1)) \mid x(.) \text { is a (nonrelaxed) feasible } \\
\text { state trajectory such that } \left.\|x(.)-\bar{x}(.)\|_{L^{\infty}} \leq \epsilon\right\}
\end{array}
$$

Then conditions (i)-(iv) above are satisfied for some choice of multipliers $(p(),. \mu(),. \lambda)$ such that $\lambda=0$.

Since optimal control problems without state constraints are special cases of problems with state constraints, the example of section 3 serves to illustrate the distinctions highlighted in the preceeding two theorems.

5. Proof of the theorems. In this section we provide proofs of the theorems stated in earlier sections. It is sufficient to prove Theorems 4.1 and 4.2 , because Theorems 2.1 and 2.2 are simply special cases, in which the state constraint " $h(t, x(t)) \leq 0$ " is dropped from the formulation. To be precise, to prove Theorems 2.1 and 2.2 for the state constraint-free problem $(P)$, we apply Theorems 4.1 and 4.2 to a state constrained problem $\left(P_{S}\right)$, in which the state constraint functional $h(.,$.$) is chosen to be$ $h(.,.) \equiv-1$. We recover precisely the assertions of Theorems 2.1 and 2.2 , because the new state constraint does not alter the sets of feasible state trajectories or relaxed feasible state trajectories, and because the state constraint measure multiplier can be taken to be the zero measure, since (for the above choice of $h(.,$.$) ) it always has an$ empty support set.

Theorem 4.1 was stated first because it is of greater interest than Theorem 4.2. We prove the theorems in reverse order, however, because the proof of Theorem 4.1 builds on that of Theorem 4.2 .

We may assume, without loss of generality, that the functions $c($.$) and k($.$) in$ (H2) are essentially bounded; we may take them then to have constant values, written $c$ and $k$. This is because, if not, we can introduce a change of time variable which renders them essentially bounded; we then prove the stated assertions for the transformed problem, which imply the same assertions for the original problem (cf. the analysis in [3, section 2.4]). This reduction procedure is standard in the derivation of necessary conditions; its use in the present context is also justified because, here, "local" minimizers are interpreted in the $L^{\infty}$ sense, and $L^{\infty}$ distances between state trajectories (or relaxed state trajectories) are unaffected by time reparameterization. Define

$$
\mathcal{S}:=\left\{x(.) \in W^{1,2} \mid \dot{x}(t) \in F(t, x(t)),\|x(.)-\bar{x}(.)\|_{L^{\infty}} \leq \bar{\epsilon}\right\}
$$


Here, $\bar{\epsilon}$ is the constant of hypothesis (H2). Notice that state trajectories $x($.$) such$ that $\|x(.)-\bar{x}(.)\|_{L^{\infty}} \leq \bar{\epsilon}$ define elements in $W^{1,2}$, because (H2) is assumed to be satisfied with $c\left(\right.$.) replaced by the constant $c$. Here, $W^{1,2}$ is the space of absolutely continuous $\mathbb{R}^{n}$-valued functions with square integrable derivatives, and inner product

$$
\left\langle\left(x(.), x^{\prime}(.)\right)\right\rangle=x(0) \cdot x^{\prime}(0)+\int_{0}^{1} \dot{x}(t) \cdot \dot{x}^{\prime}(t) d t .
$$

Lemma 5.1. There exists a monotone increasing function $\eta():.[0, \infty) \rightarrow[0, \infty)$ such that $\lim _{s \downarrow 0} \eta(s)=0$ and

$$
\left\|x^{\prime}(.)-x(.)\right\|_{L^{\infty}} \leq \eta\left(\left\|x^{\prime}(.)-x(.)\right\|_{L^{2}}\right)
$$

for any $x(),. x^{\prime}(.) \in \mathcal{S}$.

Proof. Take any $x(),. x^{\prime}(.) \in \mathcal{S}$. Then there exists $\bar{t} \in[0,1]$ such that

$$
\left.\| x^{\prime}(.)-x(.)\right) \|_{L^{\infty}}=\left|x^{\prime}(\bar{t})-x(\bar{t})\right| .
$$

Assume $\bar{t} \leq 1 / 2$. (The modifications to the analysis to deal with the other case, $\bar{t}>1 / 2$, are obvious.) It follows from hypothesis (H2) (c(.) is replaced by $c$, remember) that, for all $t$ 's in some subinterval of $I \subset[0,1]$ of length at least

$$
\left(\frac{1}{2 c}\left|\left(x-x^{\prime}\right)(\bar{t})\right|\right) \wedge \frac{1}{2}
$$

we have $\left|\left(x^{\prime}-x\right)(t)\right|^{2} \geq \frac{1}{4}\left|\left(x^{\prime}-x\right)(\bar{t})\right|^{2}$. It follows that

$$
\left\|\left(x^{\prime}-x\right)(.)\right\|_{L^{2}}^{2} \geq \frac{1}{4}\left\|\left(x^{\prime}-x\right)(.)\right\|_{L^{\infty}}^{2} \times\left[\left(\frac{1}{2 c}\left\|\left(x^{\prime}-x\right)(.)\right\|_{L^{\infty}}\right) \wedge \frac{1}{2}\right] .
$$

But then (5.2) is true, when $\eta($.$) is taken to be the continuity modulus$

$$
\eta(\sigma):=\left[(8 c)^{\frac{1}{3}} \sigma^{\frac{2}{3}}\right] \vee\left[(8)^{\frac{1}{2}} \sigma\right] .
$$

Proof of Theorem 4.2.

(a) Take a sequence $\delta_{i} \downarrow 0$. We know from the relaxation theorem (see, e.g., $\left[9\right.$, Thm. 2.7.2]) that there exists a sequence of state trajectories $y_{i}($.$) such that, for$ each $i$,

$$
\left\|y_{i}(.)-\bar{x}(.)\right\|_{L^{\infty}} \leq \delta_{i} .
$$

Take a sequence $\epsilon_{i} \downarrow 0$ such that

$$
\epsilon_{i}>\sqrt{2} k_{g} \delta_{i}
$$

for all $i$, where $k_{g}$ is a Lipschitz constant for $g(.,$.$) on a neighborhood of (\bar{x}(0), \bar{x}(1))$. For each $i$ define the function $J_{i}: \mathcal{S} \rightarrow \mathbb{R}$,

$$
\begin{array}{r}
J_{i}(x(.)):=\left(\left(g(x(0), x(1))-g(\bar{x}(0), \bar{x}(1))+\epsilon_{i}\right) \vee d_{C}(x(0), x(1)) \vee \max _{t \in[0,1]} h(t, x(t))\right) \\
+\int_{0}^{1}\left(x(t)-y_{i}(t)\right)^{2} d t .
\end{array}
$$

Copyright $@$ ㅇ by SIAM. Unauthorized reproduction of this article is prohibited. 
Now consider the optimization problem

$$
\text { minimize } J_{i}(x(.)) \text { over } x(.) \in \mathcal{S} \text {. }
$$

The function $J_{i}($.$) is continous and bounded below on the closed subset \mathcal{S}$ of the Hilbert space $W^{1,2}$. The extension of this function to all $W^{1,2}$, obtained by setting its value to $+\infty$ outside $\mathcal{S}$, is lower semicontinous and bounded below. Now apply Stegall's variational principle (see, e.g., [4, Thm. 4.2]) to the extended function. This tells us that there exist a sequence $\left\{\left(\alpha_{i}, \beta_{i}().\right)\right\}$ in $\mathbb{R}^{n} \times L^{2}$ and a sequence of elements $\left\{x_{i}\right\}$ in $\mathcal{S}$ such that, for each $i$,

$$
\left|\alpha_{i}\right|<\epsilon_{i} \text { and }\left\|\beta_{i}(.)\right\|_{L^{2}}<\epsilon_{i},
$$

and $x_{i}($.$) is a minimizer for the problem$

$$
\text { minimize } J_{i}(x(.))+\alpha_{i} \cdot x(0)+\int_{0}^{1} \beta_{i}(t) \cdot \dot{x}(t) d t \quad \text { over } \quad x(.) \in \mathcal{S} .
$$

But since $x_{i}($.$) must have cost lower than that of y_{i}($.$) ,$

$$
\begin{aligned}
\int_{0}^{1}\left(x_{i}(t)-y_{i}(t)\right)^{2} d t \leq & J_{i}\left(x_{i}(.)\right) \\
\leq & J_{i}\left(y_{i}(.)\right)+\alpha_{i} \cdot\left(y_{i}(0)-x_{i}(0)\right)+\int_{0}^{1} \beta_{i}(t) \cdot\left(\dot{y}_{i}(t)-\dot{x}_{i}(t)\right) d t \\
\leq & \left(\epsilon_{i} \vee d_{C}\left(y_{i}(0), y_{i}(1)\right) \vee \max _{t \in[0,1]} h\left(t, y_{i}(t)\right)\right)+\epsilon_{i} \times \bar{\epsilon} \\
& +\epsilon_{i} \times\left(\int_{0}^{1}\left|\dot{y}_{i}(t)-\dot{x}_{i}(t)\right|^{2} d t\right)^{\frac{1}{2}} \leq K \epsilon_{i}
\end{aligned}
$$

for some constant $K$ which does not depend on $i$. (We have made use of (5.3), (5.4), (5.5), and the Schwarz inequality.)

It follows from (5.7), Lemma 5.1, and the triangle inequality that, for each $i$,

$$
\left\|x_{i}(.)-\bar{x}(.)\right\|_{L^{\infty}} \leq\left\|x_{i}(.)-y_{i}(.)\right\|_{L^{\infty}}+\left\|y_{i}(.)-\bar{x}(.)\right\|_{L^{\infty}} \leq \eta\left(K \epsilon_{i}\right)+\epsilon_{i} .
$$

We conclude that $\left\|x_{i}(.)-\bar{x}(.)\right\|_{L^{\infty}} \rightarrow 0$ as $i \rightarrow \infty$. Now write

$$
L(t, x, v)=\left(x-y_{i}(t)\right)^{2}+\beta_{i}(t) \cdot v .
$$

Noting that $\operatorname{co}\{(e, L(t, x, e)) \mid e \in F(t, x)\}$ is $\{(e, L(t, x, e)) \mid e \in \operatorname{co} F(t, x)\}$ (this follows from the fact that $e \rightarrow L(t, x, e)$ is an affine function) and appealing once again to the relaxation theorem, we see that, for $i$ sufficiently large (in which case $\left.\left\|x_{i}(.)-\bar{x}(.)\right\|_{L^{\infty}}<\bar{\epsilon}\right), x_{i}($.$) continues to be a minimizer for problem (5.6) when, in$ the definition of $\mathcal{S}, \operatorname{co} F(t, x)$ is substituted in place of $F(t, x)$.

The preceding minimizing property of $x_{i}($.$) can be expressed in the following$ optimal control theoretic terms: for each $i$ sufficiently large,

$$
\left(x_{i}(.), b_{i}(.) \equiv t \rightarrow \int_{0}^{t} L\left(s, x_{i}(s), \dot{x}_{i}(s)\right) d s, c_{i}(.) \equiv \max _{s \in[0,1]} h\left(s, x_{i}(s)\right)\right)
$$

Copyright $@$ by SIAM. Unauthorized reproduction of this article is prohibited. 
is a strong local minimizer for

$$
\left\{\begin{array}{l}
\operatorname{minimize} \tilde{g}(x(0), x(1), c(1), b(1))+\alpha_{i} \cdot x(0) \\
\text { over }(x(.), b(.), c(.)) \in W^{1,1} \text { satisfying } \\
(\dot{x}(t), \dot{b}(t), \dot{c}(t)) \in \tilde{F}_{i}(t, x(t)) \quad \text { a.e. } \\
h(t, x(t))-c(t) \leq 0 \quad \text { for all } t \in[0,1] \\
b(0)=0
\end{array}\right.
$$

in which

$$
\tilde{g}\left(x_{0}, x_{1}, b_{1}, c\right)=\left(\left(g\left(x_{0}, x_{1}\right)-g\left(y_{i}(0), y_{i}(1)\right)+\epsilon_{i}\right) \vee d_{C}\left(x_{0}, x_{1}\right) \vee c\right)+b_{1}
$$

and $\tilde{F}_{i}(.,):.[0,1] \times \mathbb{R}^{n} \leadsto \mathbb{R}^{n} \times \mathbb{R} \times \mathbb{R}$ is the convex valued multifunction

$$
\tilde{F}_{i}(t, x)=\{(v, L(t, x, v), 0): v \in \operatorname{co} F(t, x)\} .
$$

Notice that

$$
\left(g\left(x_{i}(0), x_{i}(0)\right)-g\left(y_{i}(0), y_{i}(1)\right)+\epsilon_{i}\right) \vee d_{C}\left(x_{i}(0), x_{i}(1)\right) \vee c>0
$$

since, otherwise, $x_{i}($.$) would be a feasible F$-trajectory satisfying $\left\|x_{i}(.)-\bar{x}(.)\right\|_{L_{\infty}} \leq \epsilon$ and

$$
g\left(x_{i}(0), x_{i}(1)\right) \leq g\left(y_{i}(0), y_{i}(1)\right)-\epsilon_{i} \leq g(\bar{x}(0), \bar{x}(1))+\sqrt{2} k_{g} \delta-\epsilon_{i}<g(\bar{x}(0), \bar{x}(1)) .
$$

This is not possible in view of the minimizing properties of $\bar{x}($.$) .$

Applying the "max rule" of subdifferential calculus, taking account of the fact that limiting subdifferentials of $d_{C}(y)$ have unit length if $y \notin C$, and noting the preceding property of $x_{i}($.$) , we arrive at the limiting subdifferential estimate$

$$
\begin{aligned}
\left(p_{0}, p_{1}, r, s\right) & \in \partial \tilde{g}\left(x_{i}(0), x_{i}(1), b_{i}(1), c_{i}\right) \quad \text { implies } \\
\left(p_{0}, p_{1}\right) & \in \lambda \partial g\left(x_{i}(0), x_{i}(1)\right)+\rho\left(\partial d_{C}\left(x_{i}(0), x_{i}(1)\right) \cap\left\{\xi^{\prime}|| \xi^{\prime} \mid=1\right\}\right), \\
r & =1, \text { and } s=1-(\lambda+\rho),
\end{aligned}
$$

in which $\lambda$ and $\rho$ are positive numbers such that $\lambda+\rho \leq 1$.

Writing the multipliers associated with the $(x, b, c)$ components as $(p, r, s)$, we can express the Hamiltonian for the multifunction $\tilde{F}_{i}(t, x)$ as

$$
\tilde{H}_{i}(t, x, p, r):=H\left(t, x, p+r \beta_{i}(t)\right)+r\left(x-y_{i}(t)\right)^{2},
$$

where $H(., .,$.$) is the Hamiltonian for F(.,$.$) . Notice once again the critical role of now$ using a perturbation term $+\int_{0}^{1} b(t) \cdot \dot{x}(t) d t$, which is affine in the velocity, to permit this simple representation of the modified Hamiltonian.

The conditions for the application of the Hamiltonian inclusion conditions (see, e.g., [9, Thm. 10.4.1]) are satisfied. Owing to the absence of right endpoint constraints and the special nature of the state constraint (it results from a reformulation of a problem with a "min-max" term in the cost), the conditions apply in normal form. They assert the existence of $\left(p_{i}(),. r_{i}(),. s_{i}().\right) \in W^{1,1}$, an element $\mu_{i}(.) \in N B V^{+}[0,1]$, a $\mu_{i}$-integrable function $m_{i}(),. \xi_{i} \in \mathbb{R}^{n} \times \mathbb{R}^{n}$, and nonnegative numbers $\lambda_{i}$ and $\rho_{i}$ satisfying $\lambda_{i}+\rho_{i} \leq 1$, such that

(i) $\lambda_{i}+\left|\xi_{i}\right|+\int_{[0,1]} \mu_{i}(d s)=1$, 
$(\text { ii })^{\prime}\left(-\dot{p}_{i}(t), \dot{x}_{i}(t), \dot{b}_{i}(t)\right) \in \operatorname{co} \partial_{x, p, r} \tilde{H}_{i}\left(t, x_{i}(t), p_{i}(t)+\int_{[0, t]} m_{i}(s) \mu_{i}(d s), r_{i}(t)=1\right)$ a.e.,

(iii) $^{\prime}(\mathrm{a})\left(p_{i}(0),-\left(p_{i}(1)+\int_{[0,1]} m_{i}(s) \mu_{i}(d s)\right)\right) \in \lambda_{i} \partial g\left(x_{i}(0), x_{i}(1)\right)+\left(\alpha_{i}, 0\right)+\xi_{i}$,

(iii) $^{\prime}(\mathrm{b}) \xi_{i} \in N_{C}\left(x_{i}(0), x_{i}(1)\right)$,

(iv) $m_{i}(t) \in \partial_{x}^{>} h\left(t, x_{i}(t)\right) \quad \mu_{i}$-a.e. $t \in[0,1]$.

Taking note of the representation (5.10), we deduce from condition (ii) ${ }^{\prime}$ that $\left(p_{i}(),. x_{i}().\right)$ satisfies

(ii) $^{\prime}(\mathrm{a}):\left(-\dot{p}_{i}(t), \dot{x}_{i}(t)\right)$

$$
\begin{aligned}
\in \bigcup_{|\beta| \leq\left|\beta_{i}(t)\right|}\left\{\operatorname{co} \partial_{x, p, r} H\left(t, x_{i}(t), p_{i}(t)+\int_{[0, t]} m_{i}(s) \mu_{i}(d s)+\beta\right)\right\} & +\{0\} \times\left(2\left|x_{i}(t)-y_{i}(t)\right| B\right) \quad \text { a.e. }
\end{aligned}
$$

It can be deduced from (i) $)^{\prime}$, (ii)' (a), (iii)'((a) and (b)), and the fact that $\beta_{i}(.) \rightarrow 0$ strongly in $L^{2}$, that $\left\{\left(x_{i}(),. p_{i}().\right)\right\}$ is a uniformly bounded sequence. Furthermore, the sequence $\left\{\left(\dot{x}_{i}(),. \dot{p}_{i}().\right)\right\}$ is equi-integrable. It follows that, along a subsequence (we do not relabel), $\left(x_{i}(),. p_{i}().\right) \rightarrow(\bar{x}(),. p()$.$) uniformly and \left(\dot{x}_{i}(),. \dot{p}_{i}().\right) \rightarrow(\dot{\bar{x}}(),. p()$. weakly in $L^{1}$ for some $p(.) \in W^{1,1}$. We can arrange, by further subsequence extraction, that $\lambda_{i} \rightarrow \lambda, \xi_{i} \rightarrow \xi$, and $\mu_{i} \rightarrow \mu$ in the weak* $N B V^{+}$topology, for some $\lambda \geq 0$, $\xi \in N_{C}(\bar{x}(0), \bar{x}(1))$, and $\mu \in N B V^{+}$. A convergence analysis similar to that in [9, section 9.3] permits us to pass to the limit in the preceding relations, thereby obtaining

(i) $\lambda+|\xi|+\int_{[0,1]} \mu(d s)=1$,

(ii) $(-\dot{p}(t), \dot{\bar{x}}(t)) \in \operatorname{co} \partial_{x, p} H\left(t, \bar{x}(t), p(t)+\int_{[0, t]} m(s) \mu(d s)\right) \quad$ a.e.,

(iii) (a) $\left(p(0),-\left(p(1)+\int_{[0,1]} m(s) \mu(d s)\right)\right) \in \lambda \partial g(\bar{x}(0), \bar{x}(1))+\xi$,

(iii) (b) $\xi \in N_{C}(\bar{x}(0), \bar{x}(1))$,

(iv) $m(t) \in \partial_{x}^{>} h(t, \bar{x}(t)) \quad \mu$-a.e. $t \in[0,1]$.

The assertions of the theorem (part (a)) follow since, if $(\lambda, \mu())=.(0,0)$, then $\xi \neq 0$. It follows that, in this case, $p() \neq$.0 .

(b) Take a sequence $\delta_{i} \downarrow 0$. By the relaxation theorem, there exists a sequence of state trajectories $y_{i}($.$) such that$

$$
\left\|y_{i}(.)-\bar{x}(.)\right\|_{L^{\infty}} \leq \delta_{i}
$$

for each $i$. Take $\mathcal{S}$ as in (5.1), but now define the function $J_{i}: \mathcal{S} \rightarrow \mathbb{R}$ to be

$$
J_{i}(x(.)):=\left(d_{C}(x(0), x(1)) \vee \max _{t \in[0,1]} h(t, x(t))\right)+\int_{0}^{1}\left|x(t)-y_{i}(t)\right|^{2} d t .
$$

Now consider the optimization problem

$$
\text { minimize } J_{i}(x(.)) \text { over } x(.) \in \mathcal{S} \text {. }
$$

As in the proof of part (a) of the theorem, we deduce from Stegall's theorem that there exist a sequence $\left\{\left(\alpha_{i}, \beta_{i}().\right)\right\}$ in $\mathbb{R}^{n} \times L^{2}$ and a sequence of elements $\left\{x_{i}\right\}$ in $\mathcal{S}$ such that, for each $i$,

$$
\left|\alpha_{i}\right|<\epsilon_{i} \quad \text { and } \quad\left\|\beta_{i}(.)\right\|_{L^{2}}<\epsilon_{i}
$$

and $x_{i}($.$) is a minimizer for the problem$

$$
\text { minimize } J_{i}(x(.))+\alpha_{i} \cdot x(0)+\int_{0}^{1} \beta_{i}(t) \cdot \dot{x}(t) d t \quad \text { over } \quad x(.) \in \mathcal{S} .
$$

Invoking Lemma 5.1, we can show that

$$
\left\|x_{i}(.)-\bar{x}(.)\right\|_{L^{\infty}} \rightarrow 0 \text { as } i \rightarrow \infty .
$$

Copyright (c) by SIAM. Unauthorized reproduction of this article is prohibited. 
It follows from the hypotheses of part (b) of the theorem that, for $i$ sufficiently large, $x_{i}($.$) cannot be feasible. This implies$

$$
d_{C}\left(x_{i}(0), x_{i}(1)\right) \vee \max _{t \in[0,1]} h\left(t, x_{i}(t)\right)>0 .
$$

As in part (a), we can conclude from these properties that, for $i$ sufficiently large, $\left(x_{i}(),. b_{i}(),. c_{i}().\right)$, given by $(5.8)$, is a strong local minimizer for the

$$
\left\{\begin{array}{l}
\operatorname{minimize} \tilde{g}^{(1)}(x(0), x(1), c(1), b(1))+\alpha_{i} \cdot x(0) \\
\text { over }(x(.), b(.), c(.)) \in W^{1,1} \text { satisfying } \\
(\dot{x}(t), \dot{b}(t), \dot{c}(t)) \in \tilde{F}_{i}(t, x(t)) \text { a.e. } \\
h(t, x(t))-c(t) \leq 0 \quad \text { for all } t \in[0,1] \\
b(0)=0
\end{array}\right.
$$

in which $\tilde{F}_{i}(.,$.$) is given by (5.9)$ and

$$
\tilde{g}^{(1)}\left(x_{0}, x_{1}, b_{1}, c\right):=\left(\left(d_{C}\left(x_{0}, x_{1}\right)\right) \vee c\right)+b_{1} .
$$

Using the nondegeneracy property (5.11), the max rule of subdifferential calculus, and the fact that " $\xi \in d_{C}(z)$ and $z \notin C$ " implies " $|\xi|=1$ " (see, e.g., [9, Lemma 7.2.1]), we can show that

$$
\begin{aligned}
\left(p_{0}, p_{1}, r, s\right) & \in \partial \tilde{g}^{(1)}\left(x_{i}(0), x_{i}(1), b_{i}(1), c_{i}\right) \text { implies } \\
\left(p_{0}, p_{1}\right) & \in \rho\left(\partial d_{C}\left(x_{i}(0), x_{i}(1)\right) \cap\left\{\xi^{\prime}|| \xi^{\prime} \mid=1\right\}\right), r=1, \text { and } s=1-\rho .
\end{aligned}
$$

in which $\rho \in[0,1]$.

Now apply the Hamiltonian inclusion conditions (which are valid here in normal form). They assert the existence of $\left(p_{i}(),. r_{i}(),. s_{i}().\right) \in W^{1,1}$, an element $\mu_{i}(.) \in$ $N B V^{+}[0,1]$, a $\mu_{i}$-integrable function $m_{i}(),. \xi_{i} \in \mathbb{R}^{n} \times \mathbb{R}^{n}$, and $\rho_{i} \in[0,1]$, such that

(i) $)^{\prime}\left|\xi_{i}\right|+\int_{[0,1]} \mu_{i}(d s)=1$,

(ii) $^{\prime}\left(-\dot{p}_{i}(t), \dot{x}_{i}(t), \dot{b}_{i}(t)\right) \in \operatorname{co} \partial_{x, p, r} \tilde{H}_{i}\left(t, x_{i}(t), p_{i}(t)+\int_{[0, t]} m_{i}(s) \mu_{i}(d s), r_{i}(t)=1\right)$ a.e.,

(iii) $^{\prime}$ (a) $\left(p_{i}(0),-\left(p_{i}(1)+\int_{[0,1]} m_{i}(s) \mu_{i}(d s)\right)\right)=\left(\alpha_{i}, 0\right)+\xi_{i}$,

(iii)' (b) $\xi_{i} \in N_{C}\left(x_{i}(0), x_{i}(1)\right)$,

(iv) ${ }^{\prime} m_{i}(t) \in \partial_{x}^{>} h\left(t, x_{i}(t)\right) \quad \mu_{i}$-a.e. $t \in[0,1]$.

As before, we find that $\left\{\left(x_{i}(),. p_{i}().\right)\right\}$ is uniformly bounded and $\left\{\left(\dot{x}_{i}(),. \dot{p}_{i}().\right)\right\}$ equi-integrable. Restricting attention to subsequences then, we can find $\left(x_{i}(),. p_{i}().\right) \rightarrow$ $(\bar{x}(),. p()$.$) uniformly, \left(\dot{x}_{i}(),. \dot{p}_{i}().\right) \rightarrow(\dot{\bar{x}}(),. p()$.$) weakly in L^{1}, \xi_{i} \rightarrow \xi$, and $\mu_{i} \rightarrow \mu$ in the weak* $N B V^{+}$topology, for some $\xi \in N_{C}(\bar{x}(0), \bar{x}(1))$ and $\mu \in N B V^{+}$. In the limit as $i \rightarrow \infty$ we obtain

(i) $|\xi|+\int_{[0,1]} \mu(d s)=1$,

(ii) $(-\dot{p}(t), \dot{\bar{x}}(t)) \in \operatorname{co} \partial_{x, p} H\left(t, \bar{x}(t), p(t)+\int_{[0, t]} m(s) \mu(d s)\right) \quad$ a.e.,

(iii) $\left(p(0),-\left[p(1)+\int_{[0,1]} m(s) \mu(d s)\right]\right)=\xi \quad$ and $\quad \xi \in N_{C}(\bar{x}(0), \bar{x}(1))$,

(iv) $m(t) \in \partial_{x}^{>} h(t, \bar{x}(t)) \quad \mu$-a.e. $t \in[0,1]$.

The assertions of part (b) follow.

Proof of Theorem 4.1. Part (a) of the theorem is well known (see, e.g., [9, section 10.4]). We restrict attention to the proof of part (b). Take a sequence $\epsilon_{i} \downarrow 0$. Under the hypotheses of part (b) of the theorem there exists a sequence relaxed feasible state trajectories $\left\{x_{i}().\right\}$ such that, for each $i$,

$$
\left\|x_{i}(.)-\bar{x}(.)\right\|_{L^{\infty}} \leq \epsilon_{i}
$$

Copyright (C) by SIAM. Unauthorized reproduction of this article is prohibited. 
and

$$
g\left(x_{i}(0), x_{i}(1)\right)<g(\bar{x}(0), \bar{x}(1)) .
$$

For $i$ sufficiently large, the relaxed trajectory $x_{i}($.$) satisfies the hypotheses of Theo-$ rem 4.2(b). We now apply Theorem 4.2 (or, to be more precise, the slightly stronger form stated as conditions (i)-(iv) at the end of the proof of Theorem 4.2). We conclude that, for each $i$, there exist $p_{i}(.) \in W^{1,1}$, a measure $\mu_{i} \in N B V^{+}[0,1]$, and a $\mu_{i}$-integrable function $\gamma_{i}($.$) and \xi_{i} \in \mathbb{R}^{n} \times \mathbb{R}^{n}$ such that:

(i) $)^{\prime}\left|\xi_{i}\right|+\int_{[0,1]} \mu_{i}(d s)=1$,

(ii) $)^{\prime}\left(-\dot{p}_{i}(t), \dot{x}_{i}(t)\right) \in \operatorname{co} \partial H\left(t, x_{i}(t), p_{i}(t)+\int_{[0, t]} \gamma_{i}(s) \mu_{i}(d s)\right) \quad$ a.e.,

(iii) $^{\prime}\left(p_{i}(0),-\left[p_{i}(1)+\int_{[0,1]} \gamma_{i}(s) \mu_{i}(d s)\right]\right)=\xi \quad$ and $\quad \xi_{i} \in N_{C}\left(x_{i}(0), x_{i}(1)\right)$,

(iv) $\gamma_{i}(t) \in \partial_{x}^{>} h\left(t, x_{i}(t)\right) \quad \mu_{i}$-a.e. $t \in[0,1]$.

Carrying out a similar convergence analysis to those described above, we find that there exist $p(.) \in W^{1,1}, \xi \in \mathbb{R}^{n} \times \mathbb{R}^{n}, \mu(.) \in N B V^{+}$, and a $\mu$-integrable function $\gamma($. such that

(i) $|\xi|+\int_{[0,1]} \mu(d s)=1$,

(ii) $(-\dot{p}(t), \dot{\bar{x}}(t)) \in \operatorname{co} \partial_{x, p} H\left(t, \bar{x}(t), p(t)+\int_{[0, t]} \gamma(s) \mu(d s)\right)$ a.e.,

(iii) $\left(p(0),-\left[p(1)+\int_{[0,1]} \gamma(s) \mu(d s)\right]\right)=\xi \quad$ and $\quad \xi \in N_{C}(\bar{x}(0), \bar{x}(1))$,

(iv) $\gamma(t) \in \partial_{x}^{>} h(t, \bar{x}(t)) \quad \mu$-a.e. $t \in[0,1]$.

The theorem is proved.

\section{REFERENCES}

[1] M. S. Aronna, J. F. Bonnans, And P. Martinon, A well-posed shooting algorithm for optimal control problems with singular arcs, J. Optim. Theory Appl., 158 (2013), pp. 419-459.

[2] F. H. Clarke, Optimization and Nonsmooth Analysis, Wiley-Interscience, New York, 1983.

[3] F. H. Clarke, Necessary conditions in dynamic optimization, Mem. Amer. Math. Soc., 173 (2005), 816.

[4] F. H. Clarke, Y. S. Ledyaev, R. J. Stern, and P. R. Wolenski, Nonsmooth Analysis and Control Theory, Grad. Texts Math. 178, Springer Verlag, New York, 1998.

[5] A. Ioffe, Euler Lagrange and Hamiltonian formalisms in dynamic optimization, Trans. Amer. Math. Soc., 349 (1997), pp. 2871-2900.

[6] A. Ioffe, Optimal control of differential inclusions: New developments and open problems, in Proceedings of the 41st IEEE Conference on Decision and Control, IEEE, Piscataway, NJ, 2002, pp. 3127-3132.

[7] T. T. Rockafellar and J.-B. Wets, Variational Analysis, Grundlehren Math. Wiss. 317, Springer Verlag, New York, 1998

[8] R. B. VINTER, The Hamiltonian inclusion for nonconvex velocity sets, SIAM J. Control Optim., 52 (2014), pp. 1237-1250.

[9] R. B. Vinter, Optimal Control, Birkhäuser, Boston, 2000.

[10] J. WARGA, Normal control problems have no minimizing strictly original solutions, Bull. Amer. Math. Soc., 77 (1971), pp. 625-628.

[11] J. WARga, Optimal Control of Differential and Functional Equations, Academic Press, New York, 1972.

[12] J. WARGA, Controllability, extremality, and abnormality in nonsmooth optimal control, J. Optim. Theory Appl., 41 (1983), pp. 239-260.

[13] J. WARGA, Optimization and controllability without differentiability assumptions, SIAM J. Control Optim., 21 (1983), pp. 837-855. 